\title{
A MORPHEME-BASED MODEL OF NONSENTENTIAL UTTERANCE PRODUCTION
}

\author{
SHINJI IDO \\ Tohoku University / University of Sydney
}

\section{A B S T RAC T}

The present study is an attempt to account for non-sentential utterance (NSU) production without assuming the existence of a 'syntactically full sentence' for every NSU. The model for NSU production that derives from this study has the following four advantages over the popular 'constituent-omission' model: It 1) accounts for the production of NSUs that native speakers variably 'reconstruct', 2) explains why in certain contexts pro-drop cannot occur in languages that have morphologically marked subject-verb agreement 3) models the production of NSUs without devising separate production processes for 'ellipses' and 'fragments', and 4) predicts what constituents have to be present in a given NSU. It also keeps the involvement of syntax in NSU production to a minimum.

\section{[1] THE ISSUE}

In various areas of study within the discipline of linguistics, nonsentential utterances (including many of those that are referred to as elliptical sentences) are identified as 'full sentences' from which syntactic or grammatical constituents are omitted (see, e.g., Lyons $(1977,589)$, Brown and Miller (1991, 144-146), Napoli (1996, 200), Matthews (1997, 111), Bavin (2000), Malmkjær (2002, 543), Merchant (2004)). Accounts of non-sentential utterance (hereafter NSU) production where NSUs are identified as sentences with missing constituents are necessarily based on the assumption that every NSU has a corresponding 'full sentence' which native speakers 'reconstruct' with (near-)unanimous agreement. However, despite its popularity, one can find without much difficulty examples that contradict this assumption. For example, as early as 1974, Gunter $(1974,12-13)$ devised the term 'telegraphic ellipses' to refer to NSUs ('elliptical sentences' in his terminology) of which 'informants do not agree ... on the proper expansion' (ibid: 13$){ }^{1}$ The exis-

[*] Financial support for this research came in part from MEXT Grant-in-Aid for Young Scientists (B) (20720101) and the Matsushita International Foundation. I would like to thank Nayanbaatar Amarjargal, Naoya Arakawa, Joungmin Kim, Shuhrat Rahmatov, Sarvar Rahmatullaev, Kei Takahashi, Kei Yoshimoto, and the participants of the SPRIK conference 2006 for their comments, help, and criticism.

[1] For an argument concerning the indeterminacy of the contents of NSUs, see Clapp (2005, 120-121). See also Barton $(2006,19)$. The model presented in this paper postulates that an utterance may be in any form provided that $f$ 's (foci) and $m$ 's (morphemes grammatically required to accompany $f$ 's) (if there are any) are present in it and it does not contradict the proposition of the utterer. 
tence of such NSUs questions the assumption that a 'full sentence' underlies every NSU.

Recent years have seen a few separate attempts to analyze NSUs without assuming underlying full sentences. Progovac (2006) argues that full sentences are underlyingly small clauses (rather than the other way around) and that many NSUs are in fact small clauses that are not transformed into full sentences. Progovac thus argues for the feasibility of identifying NSUs as small clauses which would become full sentences through transformation. Culicover and Jackendoff (2005, chapter 7) introduce a process they call indirect licensing, through which certain phrases in NSUs are assigned semantic roles and syntactic features. This process allows NSUs to have the semantics of full sentences without actually being full sentences. Culicover and Jackendoff's analysis of NSUs hence genuinely dispenses with the notion of the underlying full sentence. These two studies aim at accounting for NSU interpretation and do not attempt an explanation of NSU production.

The present study is an attempt to account for NSU production without assuming the existence of a '(syntactically) full sentence' for every NSU. Although this study is at a somewhat preliminary stage, the model for NSU production that derives from it has the following four advantages over the popular 'constituentomission' model: 1) it accounts for the production of the sort of NSUs that Gunter calls 'telegraphic ellipses', 2) it explains why in certain contexts pro-drop cannot occur in languages that have subject-verb agreement morphology, 3) it models the production of NSUs without devising separate production processes for 'fragments' and 'ellipses', and, perhaps more significantly, 4) it predicts what constituents have to be present in a given NSU. Another characteristic of the model, which may also be an advantage over the 'constituent-omission' model, is its ability to produce NSUs with a minimum of syntax. ${ }^{2}$

The present study is also distinct from many other studies of NSUs in that it presents a model for NSU production rather than for NSU resolution, which, incidentally, has attracted enough attention among specialists to become a focus of inquiry in linguistics. Since this study models NSU production, given an appropriate set of data, it can make certain predictions about the forms of NSUs, which differentiates it from the majority of NSU studies.

The following section outlines how this model, which I tentatively call the composite model, accounts for NSU production. As will be apparent from the outline that will be presented in the following section, the composite model is capable of accounting for the production of utterances that are not syntactic constituents. (Indeed, section 4 will present an NSU that makes perfect sense despite the fact that it consists solely of bound morphemes.) In this respect too, the composite

[2] Progovac (2006) and Culicover and Jackendoff (2005) share this characteristic with the model presented here. 
model diverges radically from the majority of studies on NSUs. ${ }^{3}$

Before we proceed to the outline of the composite model, a note on the referent of the term NSU is in order. NSUs as they are identified in the present paper include not only 'elliptic utterances' but also utterances that make good sense despite their divergence from prescriptive sentence grammar, some examples of which will be dealt with in section 4 .

\section{[2] THE MODEL}

The principle on which the composite model is based is simple:

if an NSU contains foci (which are, as will be explained below, morphemes in this model) and morphemes that are grammatically required to accompany them, that NSU is sensical (i.e. makes sense).

In the remainder of this paper, I will refer to the first half of this principle, namely 'if an NSU makes sense, it necessarily contains one or more foci' as PRINCIPLE 1 and the second half, i.e. 'if an NSU makes sense, it necessarily contains morphemes that are grammatically required to accompany the focus or foci' as PRINCIPLE 2. Naturally, this second principle applies only if the grammar according to which an NSU is produced requires the focus or foci in that NSU to be accompanied by other morphemes.

The definition of 'focus' in the present study differs from current definitions in the linguistic literature. 'Focus' in the present study is, roughly speaking, the morphemic representation of the difference between discourse participants' propositions. Foci in the composite model are defined as 'morphemes that correspond with the propositional components by which a proposition differs from another proposition in the current context'. This definition, which as it is probably does not make much sense, will be explicated below with an example.

In figure 1 I present a simple schematic representation of the model in which a Turkish dialogue taken from Enç $(1986,195)$ with modification is used as an example. $f$ 's and $m$ 's in the following chart represent foci and morphemes that are grammatically required to accompany them, respectively.

[3] The composite model may hence have implications for the discussion about the categorical distinction (or lack thereof) between 'elliptical sentences' and 'sub-sentences', exemplified by Ellugardo and Stainton (2005). These implications are not unintentional but are outside the scope of the present paper. 
Mary's utterance:

(1) Herkes Ali-'yle tanış-tı.

everyone Ali-COM meet-PAST.3SG

'Everyone met Ali.'

Mary thinks that:

Everyone met Ali.

(2) $\forall x \exists e($ Meeting(e) \& Past(e) \& Agent $(e, x) \&$ Theme $(e, a))$

John wants to contradict Mary because:

John did not meet Ali.

(3) $\quad \neg \exists e($ Meeting(e) \& Past(e) \& Agent(e,j) \& Theme(e,a))

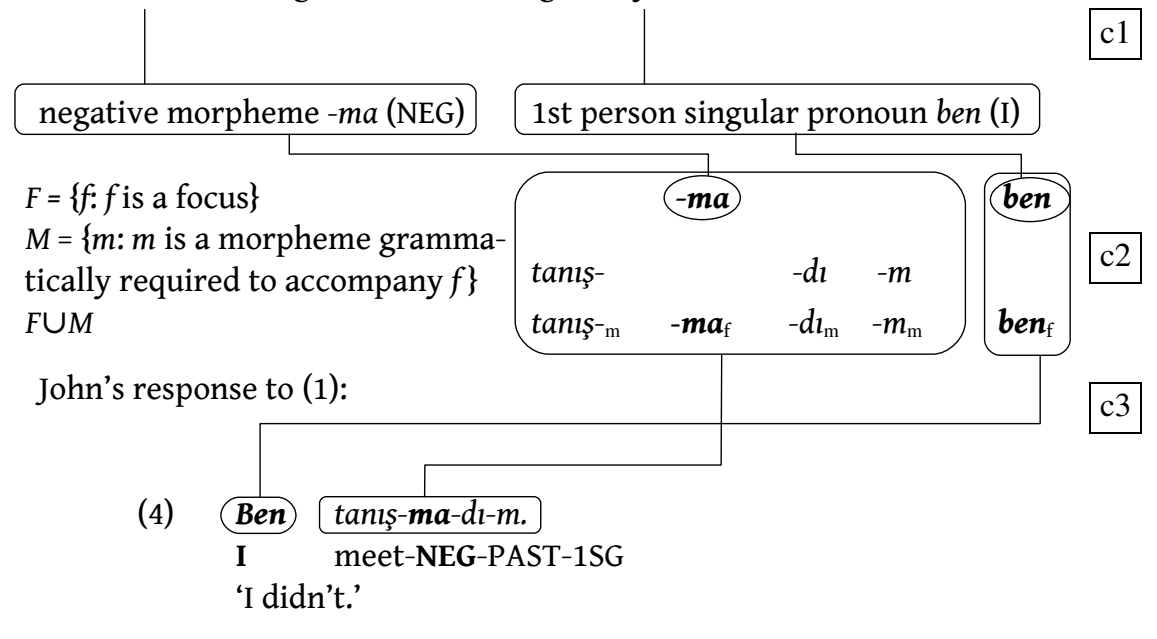

FIGURE 1: Schematic representation of the model 
Notice that (4) in figure 1 on the facing page is 'elliptic' - it is an NSU which would, in the 'constituent-omission' model, be identified as the following 'full sentence' from which Ali'yle has been omitted:

$$
\begin{aligned}
& \text { Ben Ali-'yle tanış-ma-dl-m. } \\
& \text { I Ali-COMITATIVE meet-NEGATIVE-PAST-1SG } \\
& \text { 'I did not meet Ali'. }
\end{aligned}
$$

Note also that the occurrence of the pronoun ben 'I' is obligatory in (4) despite the presence of the subject-verb agreement suffix - $m$ (1SG) - \#\# Tanışmadım is awkward as a response to (1).

As was stated above, in the composite model, 'foci' are defined as 'morphemes that correspond with the 'propositional components' by which a proposition differs from another proposition'. A more precise definition would be 'the set of morphemes onto which the difference of two sets of propositional components map'. $F$ in the following chart is the set of foci.

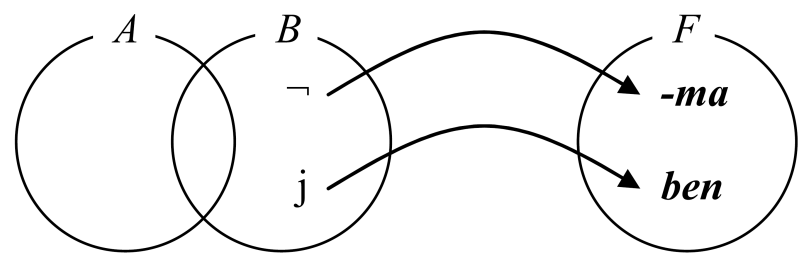

For example, if we consider (2) and (3) in figure 1 to be two sets of 'propositional components' $A$ and $B$, respectively, the difference of $B$ and $A$, or $B-A$, the elements of which are $\neg$ and $j$, corresponds to the morphemes - $m a$ and ben. - $m a$ and ben are then identified as foci. Foci are thus the morphemes that correspond with the elements of the relative complement of $A$ in $B$. (The use of the logical form as a set of 'propositional components' in this section is a temporary expedient; see section 3.)

At the time (1) is uttered, John's proposition ${ }^{4}$ (3) differs from Mary's (2) by having one connective and one individual constant, namely $\neg$ and $j$, which correspond respectively to the negative morpheme - $m a$ and the first person singular pronoun ben. These morphemes, which are called foci in this model, need to be present in (4) because Principle 1 calls for their occurrence. This explains the obligatory occurrence in (4) of the pronominal subject ben ' $\mathrm{I}$ ', whose occurrence is otherwise not obligatory. 'Pro-drop' cannot occur in (4) since Principle 1 does not allow a covert element to constitute a focus.

On the other hand, the other of the two foci, namely -ma, cannot occur in isolation and calls for the accompaniment of three morphemes, namely tanış-, $-d l$,

[4] See the next section for an explanation of the use of the term 'proposition' in this paper. 
and $-m$, the occurrence of which is required by Principle 2. I explain below why specifically tanıs-, $-d l$, and $-m$ are $m$ 's. Principle 1 calls for the occurrence of $f$ 's. Accordingly, -ma has to occur:

$-m a_{\mathrm{f}}$

NEG

In Turkish one cannot say -ma in isolation, as it is a bound morpheme. Accordingly, -ma needs to appear with some other morphemes to be grammatically acceptable. Above all, it needs a verb stem:

tanış $_{\mathrm{m}}-\boldsymbol{m} \boldsymbol{a}_{\mathrm{f}}$

meet-NEG

This means 'do not meet' in the imperative mood, which contradicts (3). Another $m$ needs to occur to render the mood of John's utterance indicative.

tanışm $_{\mathrm{m}}-\boldsymbol{m} \boldsymbol{a}_{\mathrm{f}}-d \iota_{\mathrm{m}}$

meet-NEG-PAST.3SG

This means 's/he did not meet', which also contradicts (3). Accordingly, another $m$, namely - $m$ (1SG) needs to accompany the above. Thus, if one adds morphemes to -ma until it becomes grammatically acceptable without contradicting (3), the result will be as follows. ${ }^{5}$

tanışm $-\boldsymbol{m} \boldsymbol{a}_{\mathrm{f}}-d l_{\mathrm{m}}-m_{\mathrm{m}}$

meet-NEG-PAST-1SG

Ben and tanışmadım are then linearly aligned in accordance with the minimum syntax which the alignment calls for. In the case of the production of (4), the syntactic constraint that the alignment necessitates can be as simple as 'Turkish is a verb-final language'. (If an NSU contains only one free morpheme, syntax would have no role in its production.) Syntactic constituents that Principle 1 and Principle 2 produce are often very small in number, which minimizes syntax in NSU production (likely enhancing the efficiency of utterance production).

In summary, the production of an NSU is represented in the composite model as a process in which foci $(\mathrm{fs})$ and their required accompaniments $(\mathrm{ms})$ are "put together' rather than as a process where constituents are omitted from a 'full' sentence. This model thus 1) accounts for NSU production without assuming the existence of a 'full sentence' for every NSU, 2) identifies constituents that must occur in a given NSU, 3) explains why pro-drop cannot occur in certain contexts,

[5] The morphemes added to the $f$ should be within the range of a function that has the intersection of $A$ and $B$ as its domain (see section 2). 
and 4) minimizes the role of syntax in NSU production.

\section{[2.1] Cross-linguistic validity}

The validity of the composite model is not limited to Turkish. For example, the Mongolian, Bukharan Tajik, and Japanese equivalents of (4) exhibit the same obligatory occurrence of a first person singular pronoun and a negative morpheme as was observed in Turkish (4) in the previous section. (The information structural property of each morpheme is shown in subscript.) All of these examples obey both Principle 1 and Principle 2: 'foci (f's) and morphemes that are grammatically required to accompany them ( $m$ 's) necessarily occur'.

Mary's utterance:

(1) Herkes Ali'yle tanıştı. (Turkish)

Bügd Jontoy uulzsan (biz dee). (Mongolian)

Hamma Ali kati šinos šud. (Bukharan Tajik)

Minna Arini atta (ne). (Japanese)

'Everyone met Ali'.

John's response to (1):

(4) In Turkish

$\boldsymbol{b e n}_{\mathrm{f}} \tan _{\mathrm{s}} \mathrm{s}-\boldsymbol{m} \boldsymbol{a}_{\mathrm{f}}-d_{l_{\mathrm{m}}}-m_{\mathrm{m}}$

I meet-NEG-PAST-1SGA

(4) In Mongolian ${ }^{6}$

$\boldsymbol{b i}_{\mathrm{f}}$ uul $_{\mathrm{m}}-a a_{\mathrm{m}}-\boldsymbol{g u ̈ u}_{\mathrm{f}}$

I meet-IMPERFECTIVE-NEG

(4) In Bukharan Tajik

$\boldsymbol{m a n}_{f} \boldsymbol{n} \boldsymbol{a}-{ }_{\mathrm{f}} \check{s} u d_{\mathrm{m}}-a m_{m}$

I NEG-became-1SG

(4) In Japanese

watashi $_{f^{-}} w a_{\mathrm{m}}$ aw $_{\mathrm{m}}-$ and $_{\mathrm{f}}-k a t t a_{\mathrm{m}}$

I-TOPIC meet-NEG-PAST

'I didn't'

Note that the first person singular pronoun has to occur in (4) in all of these languages, regardless of whether they utilize subject-verb agreement morphology or not.

(4) contains several $m$ 's in all of the four languages. There are, however, also languages in which (4) contains no m's, such as German, in which (4) consists only of $f$ 's:

[6] The meaning of this sentence is closer to 'I haven't met Ali' than it is to 'I didn't meet Ali'. The Mongolian translation of the latter is not used here because of the modality of regretfulness that it encodes. 
(4)

In German

$$
\begin{aligned}
& \text { Ich }_{\mathrm{f}} \text { nicht }_{\mathrm{f}} \\
& \text { I not }
\end{aligned}
$$

This German response to (1) exemplifies NSU production where there are no m's, which, unlike $f$ 's, do not occur unless the grammar according to which the utterance is produced calls for their occurrence. (The occurrence of $f$ 's, on the other hand, is obligatory in any language. $)^{7}$

Finally, let us observe the English utterance I didn't in the light of Principle 1 and Principle 2. The information structure of I didn't is analogous to that of (4) in German; I didn't has both I and not as f's. However, unlike German, not requires the accompaniment of at least one morpheme, namely the auxiliary did, thus: $\mathbf{I}_{\mathrm{f}}$ $\operatorname{did}_{\mathrm{m}}-\boldsymbol{n}^{\prime} \mathbf{t}_{\mathrm{f}} / \mathbf{I}_{\mathrm{f}} \operatorname{did}_{\mathrm{m}} \boldsymbol{n o t}_{\mathrm{f}}{ }^{8}$

[3] REPRESENTATION OF PROPOSITIONAL STRUCTURES AND QUESTIONANSWER PAIRS

This section explicates the representation of propositions used in the chart presented in the previous section. As may have been apparent from the discussion in the previous section, propositions in this model are compositional and can be decomposed into components, which are, in the outline in section 2, tentatively shown with symbols such as $\neg$ and $j$. The logical form used in (2) and (3) in figure 1 should therefore be considered representations of structured, decomposable semantic entities, for which 'propositional structures' may be a more suitable label. In this paper, however, I will continue using the term 'proposition' for the sake of simplicity.

The logical form is in fact far from adequate for the purpose of representing such propositional structures. My use of logical form in representing propositional structures is essentially an expedient, though it has an advantage, too. The logical form is appropriate for our purposes here because, unlike a number of other formalisms, such as LFG, it is impervious to syntax and grammatical relations, both of which have little place in the production of (4). ${ }^{9}$ Notwithstanding this advantage, ideally another formalism should be devised that integrates with the composite model seamlessly ${ }^{10}$, because, as an anonymous reviewer of this pa-

[7] Unless, of course, the absence of a morpheme or morphemes corresponds with an element in $B-A$ (see section 2).

[8] Note that Principle 2 does not restrict $m$ 's to bound morphemes. The auxiliary did in this example is an $m$ though it is arguably a free morpheme.

[9] In section 2, I present Mary and John's propositions as (2) and (3) because for the examples that we look at in the present paper, the 'components' of the logical form link with morphemes more or less directly.

[10] A formalism where components of a semantic representation can be mapped into a set of morphemes is particularly desirable because the logical form used in section 2 is in fact incapable of representing some types of propositions in such a way that their components can be mapped readily into a set of morphemes. 
per points out, the logical form does not fare well with other discourse contexts in which NSUs are particularly commonplace; namely discourse contexts where questions and answers take place, to which we turn now. ${ }^{11}$

In the following analysis of a question-answer pair, I tentatively use Jackendoff's Lexical Conceptual Structure 1990 as an alternative to the logical form. LCS is less of a lingua franca than first-order logic, but now that the crux of the model has been introduced, I think that the use of a notation that is of limited popularity will not render the argument here intractable. Observe the following questionanswer pair.
a. What did you eat?
b. Pilaf

The following are propositional structures of the speakers of (5a) and (5b) interpreted into LCSs. ${ }^{12}$
a. $\quad\left[\right.$ Event $\operatorname{CAUSE}\left(\left[\left[_{\text {Thing }} \mathrm{B}\right]^{\alpha}{ }_{\mathrm{A}},\left[\mathrm{GO}\left(\left[_{\text {Thing }} \mathrm{x}\right]_{\ltimes \mathrm{A}\rangle},[\mathrm{TO}[\mathrm{IN}[\mathrm{MOUTH}-\mathrm{OF}[\alpha]]]]\right)\right]\right)\right]$
b. [Event CAUSE $\left(\left[\left[_{\text {Thing }} B\right]^{\alpha}{ }_{A}\right.\right.$, $\left[\mathrm{GO}\left(\left[\left[_{\text {Thing }} \text { PILAF }\right]_{\text {(A), }}\right.\right.\right.$ TTO [IN [MOUTH-OF $\left.\left.\left.\left.\left.\left.\left.[\alpha]\right]\right]\right]\right)\right]\right)\right]$

(6a) represents, roughly, '(there exists) an event where B causes a thing $\mathrm{x}^{13}$ to go to the interior of the mouth of B'. (6b) is identical with (6a) except that it has PILAF where (6a) has an open variable $x$. Obviously, the propositional component by which (6b) differs from (6a) is PILAF. This component then finds its morphemic representation in the morpheme pilaf, which then becomes an $f$. In accordance with Principle 1, pilaf $f_{\mathrm{f}}$ has to occur in speaker B's response to (5a). Colloquial English grammar does not require any $m$ s to accompany pilaf $_{\mathrm{f}}$ and accordingly Principle 2 does not trigger any addition of $m s$ to the $\boldsymbol{p i l a f}_{\mathrm{f}}$

Turkish pilav f $_{\mathrm{f}}$ pilaf' as a response to ne ye-di-n? (what eat-PAST-2SG) 'what did you eat?' can be produced in the same way as English pila $f_{\mathrm{f}}$. So can Japanese

[11] I limit the scope of the present paper to NSUs that occur in some specific 'discourse relations' in which NSUs appear to occur routinely (e.g. correction and question-answer pair), effectively excluding discourseinitial NSUs from my discussion. The production of discourse-initial NSUs is presumably not different from that of discourse-internal NSUs except that in the former the utterer speculates on the hearer's proposition using non-linguistic clues. For instance if the hearer is a taxi driver, the utterer (B), whose proposition is [Event GO ([ [Thing B], [path TO ([place NEWTOWN])] (roughly 'B goes to Newtown') may calculate that the taxi driver's proposition is something like [Event $\mathrm{GO}$ ([Thing $\mathrm{B}]$, [Path $\mathrm{TO}$ ([place $\mathrm{X}]$ )] (roughly 'B goes to $x^{\prime}$ ) and say Newtown.

[12] These LCSs are based on the tentative analysis of the semantics of eat in Jackendoff $(1990,253)$. Details (tense etc.) that are not relevant to the argument here are omitted from these simplified LCSs.

[13] In her augmentation of LCS, Dorr $(1993,169)$ introduces such primitives as WH-MANNER, WH-THING, and WH-TIME to equip LCS with the capability to handle wh-questions ( $x$-interrogatives). The $\mathrm{x}$ here corresponds to Dorr's WH-THING. 
pirafu $\mathbf{u}_{\mathrm{f}}$ and Uzbek palov f. $^{14}$

\section{[4] DIsCUSSION}

This section recapitulates the composite model of NSU production and discusses the implications that it has for linguistic theory.

As is clear from the chart presented in section 2, the composite model comprises three main functions/mappings, namely the function that assigns certain components of a propositional structure to morphemes, which are then identified as $f$ 's (correspondence 1), the mapping between $f$ 's and $m$ 's, i.e. word-formation (correspondence 2), and the mapping of $f$ 's and $m$ 's onto a linear structure (correspondence 3). In figure 1 in section 2 , these correspondences are shown with $\mathrm{c} 1, \mathrm{c} 2$, and $\mathrm{c} 3$ in squares. The point at which the composite model departs most radically from the constituent-omission model is thus the role of syntax in NSU production. In the constituent-omission model, meaning (proposition) is mapped onto a syntactic structure, following which ellipsis takes place within that structure (see analyses in Merchant 2004). In the composite model, on the other hand, correspondences $\mathrm{c} 1$ and $\mathrm{c} 2$ restrict the role of syntax in NSU production to often very simple linear alignment of words consisting of $f$ 's and $m$ 's. The differing processes of utterance production in the constituent-omission model and that in the composite model can be schematised roughly as follows:

(7) Constituent-omission proposition $>$ syntax $>$ morphemes

Composite proposition $>$ morphemes ( $>$ word-formation $>$ syntax)

This order in which the three mappings take place means that the linear alignment of $f$ 's and $m$ 's often does not necessitate the involvement of an elaborate syntactic structure. In the case of the production of (4) in Turkish, Japanese, Mongolian, and Bukharan Tajik, there needs to be only one constraint, namely 'verbfinal', for (4) to be produced. In highly polysynthetic languages, correspondences c1 and c2 may suffice for the production of (4) because, in the composite model, syntax does not participate in NSU production unless f's and $m$ 's constitute multiple syntactic constituents. However, I would like to stress that I have no intention of defending the idea that the role of syntax is always highly restricted in NSU production. After all, the production of many of the example utterances in this paper (particularly (4) in German or English) clearly calls for the involvement of syntax. One consequence which the morpheme-based nature of the model brings about is that the model allows for the production of NSUs that do not constitute syntactic constituents. One example of such NSUs appears in the following dialogue taken

[14] Though the grammars of a number of other languages also do not require any $m s$ to accompany the focus, this does not mean that all grammars of natural languages require no $m s$ for (6b). (Indeed, the prescriptive sentence grammars of all of these languages require the occurrence of some $m$ 's for (6b).) 
from a Japanese cartoon.

(8) a. Jinsei yarinaos-e-mas-u-ka-ne.

life start-over-POTENTIAL-AHON-NONPAST-Q-SFP

'Can I start (my) life all over again?'

b. - mas $_{\mathrm{m}}-\boldsymbol{e n} \mathrm{n}$

AHON-NEG.NONPAST

'(You can)not.'

$(8 b)^{15}$ consists of two bound morphemes. ${ }^{16}$ While it is admittedly a rare occurrence for NSUs to consist exclusively of bound morphemes, such NSUs can occur and make perfect sense. Their occurrence/production hence demands a theoretical explanation. The composite model provides one such explanation: the grammar of highly colloquial Japanese requires only one $m$, namely $-m a s_{m}$ to accompany the focus $-\boldsymbol{e n}_{\mathrm{f}} \cdot{ }^{17}$

[15] The abbreviations used in this section are Q: interrogative, SFP: sentence-final particle, INTENTION: intentional mood, AHON: addressee honorifics, PROG: progressive aspect.

[16] This is by no means an isolated example of utterances consisting only of bound morphemes. An Uzbek informant provided the following constructed dialogue in which B's utterance is an NSU that consists of one bound morpheme:

(i)

$$
\begin{array}{ll}
\text { A: } & \text { u ket-ayap-ti-mi? } \\
& \text { s/he leave-PROG-3SG-Q } \\
\text { 'is s/he leaving?' } \\
\text { B: } & \text {...-moqchi } \\
& \text { INTENTION.3SG } \\
& \text { '(s/he) intends (to)'. }
\end{array}
$$

[17] The grammar of less colloquial Japanese would call for the occurrence of more m's, which would result in a longer (8b), e.g.

(8b') $\quad$ aarinaos ${ }_{\mathrm{m}}-e_{\mathrm{m}}-$ mas $_{\mathrm{m}}-\boldsymbol{e n}_{\mathrm{f}}$

start over-POTENTIAL-AHON-NEG.NONPAST

'(You) cannot start (your life) all over again'. 
Thus, one theoretical consequence that Principle 1 and Principle 2 bring about is the non-existence of 'syntactically full' sentences in the process of NSU production. ${ }^{18}$

Such a process in which NSUs are produced appears to fit the psychological reality of a number of speakers more readily than a process where components of utterances are 'omitted' or 'inaudible', though admittedly the speakers' intuitions are, in the absence of any empirical evidence, open to dispute. In summary, the composite model is based on the simple principle of the necessary minimum, namely 'for an utterance to be sensical, (only) foci and morphemes that are grammatically required to accompany them necessarily occur'. This simple principle 1) accounts for the production of NSUs that native speakers variably 'reconstruct', 2) explains why in certain contexts pro-drop cannot occur in languages that have subject-verb agreement morphology, 3) models the production of NSUs without devising separate production processes for 'ellipses' and 'fragments/subsentences', and 4) predicts what constituents have to be present in a given NSU. The composite model keeps the involvement of syntax in NSU production to a minimum, which in turn may reduce the (mental) cost of producing NSUs.

\section{REFERENCES}

Barton, E. 2006. Toward a nonsentential analysis in generative grammar. In L. Progovac, K. Paesani, E. Casielles and E. Barton (eds.), The syntax of nonsententials, pages 11-31, Amsterdam: John Benjamins.

Bavin, E. 2000. Introduction: a functional approach to ellipsis. Linguistics 38(3), 449-455.

Brown, K. and Miller, J. 1991. Syntax 2nd edition. London: Harper Collins Academic.

Clapp, L. 2005. On the interpretation and performance of non-sentential asser-

[18] For example, the ungrammaticality of (b) in the following example, which Merchant (2004, 676-705) claims to be 'expected under the ellipsis analysis, since the distribution of case morphology on DPs will be regulated by the same mechanism in both elliptical and non-elliptical contexts' (Ibid.: 679), is also expected under the present analysis, not because (b) is elliptic but because, while Yongsu is an $f$, -rul is neither an $f$ nor an $m$. This analysis is also consistent with the fact that (c), which consists of one $f$, is an acceptable answer to the question.

(i) Korean Q: $\quad \mathrm{Nu}-k a \quad k u$ chaek-ul sa-ss-ni? who-NOM this book-ACC bought

\begin{tabular}{|c|c|c|}
\hline a. & A: & $\begin{array}{l}\text { Yongsu-ka. } \\
\text { Yongsu-NOM }\end{array}$ \\
\hline b & A: & $\begin{array}{l}{ }^{*} \text { Yongsu-rul } \\
\text { Yongsu-ACC }\end{array}$ \\
\hline c & A: & $\begin{array}{l}{ }^{*} \text { Yongsu } \\
\text { Yongsu }\end{array}$ \\
\hline
\end{tabular}


tions. In R Ellugardo and R. J. Stainton (eds.), Ellipsis and nonsentential speech, pages 109-129, Dordrecht: Springer.

Culicover, P. W. and Jackendoff, R. 2005. Simpler syntax. Oxford University Press.

Dorr, B. J. 1993. The use of lexical semantics in interlingual machine translation. Machine Translation 7, 135-193.

Ellugardo, R. and Stainton, R. J. 2005. Introduction. In Ellipsis and nonsentential speech, pages 1-26, Dordrecht: Springer.

Enç, M. 1986. Topic switching and pronominal subjects in Turkish. In D. I Slobin and K Zimmer (eds.), Studies in Turkish linguistics, pages 195-208, John Benjamins.

Gunter, R. 1974. Sentences in dialog. Columbia: Hornbeam Press.

Jackendoff, R.1990. Semantic structures. Cambridge, Massachusetts: The MIT Press.

Lyons, J. 1977. Semantics volume 2. Cambridge University Press.

Malmkjær, K. 2002. The linguistics encyclopedia 2nd edition. Routledge.

Matthews, P. H. 1997. The concise Oxford dictionary of linguistics. Oxford University Press.

Merchant, J. 2004. Fragments and ellipsis. Linguistics and philosophy 27, 661-738.

Napoli, D. J. 1996. Linguistics: An Introduction. Oxford University Press.

Progovac, L. 2006. The syntax of nonsententials: Small clauses and phrases at the root. In L. Progovac, K. Paesani, E. Casielles and E. Barton (eds.), The syntax of nonsententials, pages 33-71, Amsterdam: John Benjamins.

AUTHOR CONTACT INFORMATION

Shinji Ido

Tohoku University

Research Center for Language, Brain and Cognition

41 Kawauchi, Aoba-ku, Sendai

Japan

shinji_ido@insc.tohoku.ac.jp 